\title{
MedienPädagogik
}

Zeitschrift für Theorie und Praxis der Medienbildung

Jahrbuch Medienpädagogik 2.

Zweitveröffentlichung aus: Jahrbuch Medienpädagogik 2. (2001) Opladen: Leske + Budrich. Herausgegeben von Ben Bachmair, Dieter Spanhel und Claudia de Witt.

\section{Analyse von Konzepten zum Erwerb medienpädagogischer Kompetenz \\ Folgerungen aus den Ansätzen von Dieter Baacke und Gerhard Tulodziecki}

\author{
Sigrid Blömeke
}

Die öffentliche Diskussion um die Qualifikationen von Lehrerinnen und Lehrern im Medienzusammenhang lassen den Eindruck aufkommen, sie müssten nur im Internet surfen können und mit den von ihren Schülern favorisierten Computerspielen vertraut sein und schon wäre Schule für die Herausforderungen der Informationsgesellschaft gerüstet. Entsprechend sollen in der Lehrerausbildung PC- und Internet-Führerscheine erworben werden können, alle Studierenden Email-Adressen bekommen und die Lehrenden ihre Veranstaltungen mit Computer- und Netzunterstützung durchführen, um die Lehramtsabsolventen medial fit zu machen.

In einem eigentümlichen Kontrast steht diese Diskussion zur gleichzeitigen Klage, daß allerorten - in Schule und Hochschule, Betrieb und Weiterbildung - ein Niveauverlust zu beklagen sei, daß heutige Schüler, Studierende und Auszubildende weniger könnten als frühere Generationen. Einmal abgesehen davon, daß diese Klagen vermutlich übertrieben sind: Daß hier ein Zusammenhang zur geforderten medialen Fitness bestehen könnte, wird leicht übersehen. Zeit ist schließlich keine unbegrenzte Ressource, und soll beispielsweise die Universität - und nur um sie soll es im Folgenden gehen - technische Fertigkeiten vermitteln, müssen anderswo notgedrungen Abstriche gemacht werden - u.U. mit den erwähnten Folgen.

Daß universitäre Ausbildung gerade nicht auf den Umgang mit Medien reduziert werden kann, sondern im Medienzusammenhang ganz andere Aufgaben hat, ist Thema des vorliegenden Beitrags. Systematisch wird anhand der Konzepte von Dieter Baacke und Gerhard Tulodziecki untersucht, welchen Anforderungen ein medienpädagogisch angemessen gestaltetes Lehramtsstudium zu genügen hat. (Zu dem Konzept von Dieter Spanhel vgl. den Beitrag in diesem Band.) Mit dem Ansatz des verstorbenen Bielefelder Hochschullehrers Baacke wird ein kommunikations- und sozialwissenschaftlich ausgerichtetes, mit dem Ansatz des Paderborner Hochschullehrers Tulodziecki ein allgemeindidaktisch ausgerichtetes Konzept gewählt, so daß zwei wichtige Zugänge der Erziehungswissenschaft in der Analyse Berücksichtigung finden. Dieter Baacke hat die außerschulische Medienpädagogik 
und die Arbeiten im Umkreis der Gesellschaft für Medienpädagogik und Kommunikationskultur (GMK) - der 1984 in Frankfurt/M. mit Baacke als erstem Vorsitzenden gegründeten und mit über 600 Mitgliedern bundesweit größten Fachorganisation für Medienpädagogik, Medienforschung und Medienpraxis - stark beeinflußt. Gerhard Tulodziecki steht für zahlreiche Arbeiten, die die Ausbildung kognitiver und sozial-moralischer Kompetenzen in den Vordergrund stellen. Als Sachverständiger hat er den BLKOrientierungsrahmen „Medienerziehung in der Schule“ (vgl. BLK 1995) mit geprägt, auf dessen Basis sowohl Empfehlungen der Kultusministerkonferenz als auch die meisten Richtlinien und Rahmenpläne der Länder entstanden sind. Berücksichtigt werden medienbezogene Veröffentlichungen von Baacke und Tulodziecki, ihre wichtigsten darüber hinausgehenden Werke und die umsetzungsorientierten Projekte „Medienkompetenz in der Lehrerausbildung (MeKoLa)“ an der Universität Bielefeld und „Neue Medien und Lehramtsstudium“ (im Rahmen der Initiative ,Bildungswege in der InformationsGesellschaft - BIG“) an der Universität Paderborn. Die folgende Analyse konzentriert sich vor allem auf die Frage nach dem jeweiligen Verständnis von Kompetenz, Medienkompetenz und medienpädagogischer Kompetenz als den drei zentralen Ebenen der Arbeiten der beiden Autoren. Unter Berücksichtigung der herausgearbeiteten Kritikpunkte wird eine Synthese vorgenommen, woraus Schlussfolgerungen für die Gestaltung des Lehramtsstudiums gezogen werden.

Das in diesem Beitrag gewählte klassisch hermeneutisch-interpretative Vorgehen hat seinen Bezugspunkt in einem professionstheoretisch fundierten Verständnis von Lehrerausbildung: Das Studium hat auf den späteren Beruf als Lehrerin bzw. Lehrer vorzubereiten, und zwar in wissenschaftlicher Weise. Der Ausbildungsprozess wird im Referendariat fortgesetzt, wo praktische Handlungsformen eingeübt werden. Professionalisierung bedeutet also - in Anlehnung an eine Weiterentwicklung des Ansatzes von Ulrich Oevermann - „das Hervorbringen einer besonderen Handlungsstruktur, die es ermöglicht, in der Alltagspraxis auftretende Handlungsprobleme aus der Distanz .stellvertretend' für den alltagspraktisch Handelnden wissenschaftlich reflektiert zu deuten und zu bearbeiten“ (Dewe/Ferchhoff/Radtke 1992, S. 81). Wissenschaft und Praxis stellen zwei verschiedene Perspektiven dar, aus denen ein Gegenstand - in diesem Fall der medienbezogene Lernprozess der Schülerinnen und Schüler - betrachtet wird. Um Mißverständnissen vorzubeugen, sei darauf hingewiesen, daß damit keine polyvalente Lehrerausbildung favorisiert, sondern nur ,Praxisbezug' in seiner traditionellen - eng an schulischen Unterrichtsinhalten orientierten und auf Einübung von Handlungsformen ausgerichteten - Bedeutung eine Absage erteilt wird.

Diese Einleitung abschließend sei festgehalten, daß für das Verständnis des Medienbegriffs im Rahmen dieses Beitrags im Mittelpunkt steht, daß Medien Teil eines Kommunikationsprozesses sind, sich also auf die „Wahrnehmung, Speicherung, Wiedergabe, Vervielfältigung, Übertragung und Ver- 
hen. Bei diesem ersten Definitionsschritt handelt es sich noch um einen sehr weiten Medienbegriff, der „im Grunde jedes Lebewesen und jedes Ding, welches kommunikative Inhalte transportiert“ (Sobiech 1997, S. 11) als Medium gelten lässt. In einem zweiten Schritt wird der Medienbegriff an einen Technikbegriff gekoppelt, der „,Artefakte im Sinne von gegenständlichen Gebilden, Geräten, technischen Systemen“ (Wagner 1996, S. 10) einschließt. Eine solche Definition beinhaltet sowohl die technisch unterstützte Individual- als auch jegliche Form von Massenkommunikation als den beiden Funktionen des Kommunikationsprozesses. Damit umfasst der hier verwendete Medienbegriff auf der einen Seite wie allgemein üblich beispielsweise das Buch, den Fernseher oder das Radio. Er umfasst aber auch das Telefon und das Fax, ebenso den Computer. Personale Medien wie Mimik, Gestik oder andere körpersprachliche Äußerungen der Lehrerin bzw. des Lehrers werden dagegen ausgeschlossen.

\section{Dieter Baackes Konzept einer medienpädagogisch fundierten Lehrerausbil- dung $^{1}$}

Die technische Unterstützung des Kommunikationsprozesses in Abgrenzung zu personaler Kommunikation kennzeichnet auch Baackes Medienbegriff, der im MeKoLaProjekt „,von den Printmedien (Zeitungen/Zeitschriften) über die Programm-Medien (Hörfunk/Fernsehen) bis hin zum Computer und Multimedia“ (Hugger 1995b, S. 49) reicht. Baackes strukturell vergleichbare Definition aus den 70er Jahren findet damit in späteren Arbeiten eine Erweiterung um die neuen Medien, die nunmehr sogar im „Fokus“ (Baacke 1987, S. 58) der Betrachtung stehen. Baacke zieht damit die Konsequenzen aus seiner Forderung, daß angesichts der Entwicklung der Medien eine ständige Definitionsüberprüfung des Begriffs erfolgen muss.

Eine weitere begriffliche Fundierung erfährt Baackes Konzept durch seine intensive Auseinandersetzung mit dem Kompetenzbegriff, wenn er auch den Begriff der „Medienkompetenz" entgegen der landläufigen und seiner eigenen Annahme (vgl. z.B. Aufenanger 1996a, S. 456, und Baacke 1997c, S. 26) nicht bereits 1973 in seiner Habilitation „Kommunikation und Kompetenz“ thematisiert hat, was langsam ins Bewusstsein dringt (vgl. z.B. Vollbrecht 1999, S. 13, und Kübler 1999, S. 38). Daß der Mensch kommunizieren kann, führt der ehemalige Bielefelder Hochschullehrer auf eine spezifische menschliche „Kom-

1 In der folgenden Darstellung bleibt der Text von Baacke 1999a zunächst unberücksichtigt, weil er sich in seinen Verweisen und Bezügen auf grundsätzliche erziehungswissenschaftliche Positionen deutlich von den früheren Veröffentlichungen unterscheidet. Eine Passung zu diesen ist nicht immer zu erkennen. Möglicherweise gibt Baacke hier der zunehmenden Tendenz in der bildungspolitischen Diskussion nach, vermeintlich „abgegriffene“ Begriffe durch „unverbrauchtere“ zu ersetzen, ohne die wissenschaftstheoretischen Implikationen zu bedenken. 
petenz" zurück, womit er sich an den Linguisten Noam Chomsky anlehnt (vgl. Baacke 1973, S. 100ff.). Baackes Leitbegriff der „kommunikativen Kompetenz“, den er in Abgrenzung u.a. zu Niklas Luhmanns Systemtheorie entwickelt hat, umfasst die Fähigkeit des Menschen, „potenziell situations- und aussagenadäquate Kommunikationen auszugeben und zu empfangen, ohne an Reize und von ihnen gesteuerte Lernprozesse gebunden zu sein“ (Baacke 1992b, S. 180). Die individuelle Kommunikationsfähigkeit stellt damit ein grundlegendes anthropologisches Datum dar. Das Kompetenztheorem ergänzend übernimmt Baacke von Chomsky auch das Verständnis einer „Performanz“, die eine „aktuelle Realisierung dieser (universellen; S. B.) Sprachfähigkeit in gesprochenen Sätzen“ umfaßt (Baacke 1973, S. 102).

In diesem Sinne ordnet Baacke auch den Begriff der Medienkompetenz in den Diskussionszusammenhang ein: Medienkompetenz sei eine „moderne Ausfaltung“ (Baacke 1999b, S. 19) der kommunikativen Kompetenz und bedeute, „daß jemand dann als kompetentes Lebewesen“ auch mit Medien angemessen umgehen kann, wenn bestimmte Fähigkeiten vorhanden sind, für deren Entfaltung wir freilich als Menschen .veranlagt“ sind“ (Baacke 1997b, S. 31). Genauer untersucht Baacke den Zusammenhang zwischen kommunikativer und Medienkompetenz jedoch nicht.

Ein Unterschied zu Chomsky besteht bei Baacke in der Betonung des Entwicklungsprozesses, den das Individuum durchmacht, aus dem er sogar einen Entwicklungsanspruch ableitet. Sein Leitbild ist dabei die „ideale Kommunikationsgemeinschaft“ (Apel), in der alle Menschen gleich sind und damit Verzerrungen und Störungen der Kommunikation ausgeschlossen sind. Baacke:

„Dies bedeutet, daß ,Kompetenz’ nicht, wie häufig heute, nur als Verfügung über ,skills’ (Fähigkeiten) verstanden werden darf, sondern als Begriff und Konzept einen grundlegenderen Anspruch hat, die grundlegende Eigenschaft des Menschen, ein ,kompetentes Lebewesen zu sein', zu bilden und zu fördern“ (Baacke 1994, S. 240).

Verknüpft mit Baackes gesellschaftlichen Grundannahmen ist es also pädagogische Aufgabe, „dem Menschen zu verhelfen, seine Kommunikationskompetenz für die Entscheidung zu vernünftigen Konfliktlösungen mit dem Ziel einer Aufhebung ungerechtfertigter und unfrei machender Herrschaft einzusetzen“ (Baacke 1973, S. 287). Baacke wechselt damit aus der analytischen Dimension in die (kritisch-)pädagogische mit einer normativen Zielvorgabe und bindet die zunächst nur beschreibende, also ziel- und folgenlose Feststellung einer menschlichen kommunikativen Kompetenz im Sinne von Chomsky unmittelbar an grundsätzliche individuelle und unveränderbare Menschenrechte.

Über die Sprache hinaus findet das Theorem der kommunikativen Kompetenz bei Baacke Ausweitung auf den Bereich der Wahrnehmung, da „Kommunikation nicht nur aus sprachlichen Interaktionen besteht“ (Baacke 1992b, S. 180): 
„Auch hier (im Bereich der Wahrnehmung; S. B.) werden nicht nur wahrgenommene gesehene und gehörte Gestalten isomorph aufgenommen und im internen Wahrnehmungszentrum abgebildet, sondern der Mensch kann neue Gestalten produzieren ebenso, wie er bisher nicht gehörte und gelesene Sätze bilden kann“ (Baacke 1994, S. 241).

Vor dem Hintergrund dieses Kompetenzverständnisses arbeitet Baacke sein Konzept medienpädagogischer Angebote im Lehramtsstudium aus, deren Ziel - die von Baacke so bezeichnete „Medienkompetenz“ der zukünftigen Lehrerinnen und Lehrer - sich aus mehreren Teilkompetenzen zusammensetzt. An erster Stelle steht die eigene Medienkompetenz der Lehrerinnen und Lehrer, eine „kulturelle Gesamtaufgabe für jeden Menschen“ (Baacke 1996, S. 13), die sich von kommunikativer Kompetenz dadurch unterscheidet, „daß sie nicht in face-to-face-Situationen stattfindet, sondern in der parasozialen Interaktion mit Medienbotschaften und ihren Trägern“ (Baacke 1997a, S. 54). Sie setzt sich systematisch aus zwei Bereichen zusammen: aus dem Bereich der „Vermittlung“ und aus dem Bereich der Handlung. Zu Ersterem gehört zunächst „Medienkunde“ mit wiederum zwei Elementen:

„,a) Die informative Dimension umfasst klassische Wissensbestände (wie: Was ist ein ,duales Rundfunksystem'? Wie arbeiten Journalisten? Welche Programm-Genres gibt es? Wie kann ich auswählen? Wie kann ich einen Computer für meine Zwecke effektiv nutzen? etc.);

b) die instrumentell-qualifikatorische Dimension meint die Fähigkeit, die neuen Geräte auch bedienen zu können, also z.B. das Sich-Einarbeiten in die Handhabung einer Computer-Software, das Sich-Einloggen-Können in ein Netz, usf.“ (ebd., S. 99).

Hier geht es also um Kenntnisse über Medien und Medienangebote sowie um die Fertigkeiten zur technischen Bedienung der Medien. Als „Medienkritik“ bezeichnet Baacke den zweiten Teil des „Vermittlungs“bereichs mit wiederum drei Elementen:

„a) Analytisch sollten problematische gesellschaftliche Prozesse (z.B. Konzentrationsbewegungen) angemessen erfasst werden können;

b) reflexiv sollte jeder Mensch in der Lage sein, das analytische Wissen auf sich selbst und sein Handeln anwenden zu können;

c) ethisch ist die Dimension, die analytisches Denken und reflexiven Rückbezug als sozialverantwortet abstimmt und definiert“ (ebd., S. 98).

Zur analytischen Medienkritik gehört beispielsweise auch, „die Reichweite und die Bedeutung unterschiedlicher Medien einschätzen“ (Baacke 1996, S. 12) zu können, wie Baacke an anderer Stelle ausführt. Das Element umfasst also die Fähigkeit, Medien und Medienangebote unter bestimmten Kriterien angemessen zu beurteilen.

Den zweiten großen Bereich von Medienkompetenz stellt der Handlungszusammenhang dar, den Baacke in „Mediennutzung“ und „Mediengestaltung“ ausdifferenziert. Beide Teilbereiche, in denen eine Anwendung der erworbenen Kenntnisse, Fertigkeiten und Fähigkeiten erfolgen soll, werden aktiv ausgeführt, da ,jede Art von Mediennutzung ein aktiver Vorgang ist - insofern, als auch beim Nutzer Selektions- und Wahrnehmungsvorgänge stattfinden, er also die Medienwirkung aktiv mitkonstruiert“ (Baacke 1992b, 
S. 173). Medienkompetenz setzt sich in der Baackeschen Konzeption damit aus vier Teilbereichen zusammen: Medienkunde, Medienkritik, Mediennutzung und Mediengestaltung.

Analog zum Vorgehen bei der kommunikativen Kompetenz verwendet Baacke auch Medienkompetenz nicht nur als beschreibenden Begriff, sondern bindet ihn normativ und deutet ihn als pädagogischen Auftrag, und zwar als Verantwortung dafür, daß „die Unverfügbarkeit des Subjekts sich nach dessen eigenen generativen Ausdrucksmustern entfaltet“ mit dem übergeordneten Ziel eines „Diskurses der Informationsgesellschaft“ (Baacke 1997a, S. 99f.). Dieser beinhaltet nicht nur technische und wirtschaftliche Voraussetzungen, sondern auch ein individuelles Grundrecht auf Informationsversorgung, Persönlichkeitsschutz und neue Formen politischer Kommunikation. Baacke:

„Medienkompetenz insistiert auf solchen sozialen und kulturellen Zielwerten und fordert, richtig verstanden, deren Umsetzung im Schul- und Bildungswesen, aber auch im außerschulischen Bereich ein“ (ebd., S. 102).

Auf der Basis eines solchen Verständnisses von Medienkompetenz entwickelt Baacke seine medienpädagogischen Vorstellungen, die schließlich im MeKoLa-Projekt münden. In diesem geht es letztlich darum, „den Lehrer zu befähigen, nicht nur zur ,richtigen’ Mediennutzung anzuleiten (Medienerziehung), sondern ihn auch fähig zu machen, kompetent Medien-Sprache wahrnehmen und entziffern zu können (Bildungsdimension) sowie diese Fähigkeiten auf sein eigenes Leben in der Familie, im Beruf und in sozialen Bezügen anzuwenden (Handlungsdimension)“ (Baacke 1995, S. 4f.). Die für Lehrerinnen und Lehrer notwendige ,Medienkompetenz', die es ihnen wiederum ermöglichen soll, Schülerinnen und Schülern ,Medienkompetenz’ zu vermitteln, unterscheidet Baacke sprachlich nicht voneinander. Wegen dieser Unschärfe in der Begrifflichkeit wird im Folgenden der Begriff ,Medienkompetenz' verwendet, wenn es sich um Kenntnisse, Fähigkeiten und Fertigkeiten handelt, die Schülerinnen und Schüler erwerben sollen und die einen ersten Baustein der Lehrerqualifikation darstellen. Für diese als Ganzes wird zur besseren Unterscheidung der Begriff der medienpädagogischen Kompetenz verwendet.

Die bereits im Zusammenhang der Medienkompetenz erwähnten Ebenen der Vermittlung (Medienkunde/-kritik) und der Handlung (Mediennutzung/ -gestaltung) stellen auch auf der Ebene der medienpädagogischen Kompetenz zwei Teilbereiche dar. Die Vermittlungsebene wird hier allerdings um für Lehrerinnen und Lehrer wichtige Wissensbestände wie medienpädagogische Konzepte und Modelle (z.B. Kommunikationstheorien), das Mediennutzungsverhalten von Kindern und Jugendlichen (z.B. Wissen um die Bedeutung von Kassetten oder von Werbung) oder die Kenntnis von deren Medienumgebungen (wie z.B. die Diskothek) erweitert (vgl. Hugger 1995b, S. 50 und Hugger 1995a, S. 18).

Den nach Baacke entscheidenden Unterschied zur Ebene der Medienkompetenz soll aber der dritte Teilbereich medienpädagogischer Kompetenz 
darstellen, der Bereich der sogenannten visuellen „Wahrnehmungsbildung“, in dem es um eine „Einübung in die Medienrezeption“ geht mit dem Ziel, eine Fähigkeit zu erwerben, „das Wahrnehmungsmaterial zu sortieren, das Wichtige vom Unwichtigen, die Inszenierung vom tatsächlichen Ereignis zu unterscheiden“ (Baacke 1992a, S. 41). Mit dieser Wahrnehmungsorientierung folgt Baacke auch auf der Ebene der medienpädagogischen Kompetenz der zuvor auf der Ebene der kommunikativen Kompetenz erfolgten Ausweitung des Kompetenzbegriffs. Daß Wahrnehmungsbildung — entgegen Baackes eigenen Äußerungen im Zusammenhang der Beschreibung medienpädagogischer Kompetenz - dann doch wieder nicht nur der Ebene der Lehrerinnen und Lehrer zugeschrieben wird, ist konzeptimmanent und kompetenztheoretisch folgerichtig und wird deutlich, wenn Baacke an anderer Stelle formuliert, daß angesichts der Medienentwicklung eine allgemeine Wahrnehmungsbildung immer notwendiger wird, da es heute „zu viel Wahrnehmungsmaterial (gibt, S. B.), so daß wir analog zur Informationsverschmutzung auch von einer Wahrnehmungsverschmutzung reden“ (Baacke 1995, S. 46). Zusammen mit der wachsenden Schnelligkeit der Filmschnitte führe das dazu, daß auch Kinder und Jugendliche nur noch genießen können, „wenn eine bis heute vernachlässigte Wahrnehmungsbildung ihnen das genießende Verstehen der für sie bereiteten Tele-Welten ermöglicht“ (ebd.). Wahrnehmungskompetenz ist danach also doch bereits Element grundlegender Medienkompetenz.

\section{Gerhard Tulodzieckis Konzept einer medienpädagogisch fundierten Lehrer- ausbildung}

Der Medienbegriff umfasst bei Tulodziecki - wie zuvor bei Baacke - die „technischen Medien“, mit deren Hilfe Informationen „gespeichert oder übertragen und in abbildhafter oder symbolischer Darstellung wiedergegeben werden“ (Tulodziecki/Schöpf 1992, S. 107). Konkret beinhaltet diese Definition die Printmedien, die audiovisuellen Medien und die Computer. In Bezug auf Letztere als „neue Medien“ kommt als Merkmal die Möglichkeit der Be- und Verarbeitung der Informationen hinzu (vgl. Tulodziecki 1997a, S. 37).

Der Kompetenzbegriff wird von Tulodziecki nicht näher definiert. Die theoretische Nähe seines medienpädagogischen Ansatzes (s.u.) zu einer Begriffsverwendung im Sinn einer doppelten Bedeutung von Kompetenz als komplexer Fähigkeit und normativer Zielvorgabe ist allerdings offensichtlich, wobei Tulodziecki der Realisierung der Medienkompetenz auf der Performanzebene durch einen handlungsorientierten Zugang besonderes Gewicht zuschreibt. Für die Bestimmung seiner normativen Zielvorstellungen legt Tulodziecki sein dahinter stehendes Wertesystem offen: Er greift auf Kohlbergs Theorie der sozial-moralischen Entwicklung zurück, und zwar insbesondere auf die fünfte und sechste Stufe. Letztere umfasst als Ziel für menschliches Handeln die Berücksichtigung universaler ethischer Prinzipien, 
wobei Tulodziecki einen Schwerpunkt auf Gerechtigkeit und soziale Verantwortung legt (vgl. Tulodziecki 1994a, S. 68ff.).

Entscheidende Basis für Tulodzieckis medienpädagogisches Konzept sind allgemeindidaktische Überlegungen. Unterricht soll grundsätzlich von der Leitidee eines „sachgerechten, selbstbestimmten und kreativen Handelns in sozialer Verantwortung“(ebd., S. 44) bestimmt sein. Dieser Leitidee hat auch die Entwicklung eines Konzepts zum Erwerb von Medienkompetenz in der Schule zu folgen. Tulodzieckis Ansatz eines handlungsorientierten Unterrichts berücksichtigt folgende Aspekte als Ausgangspunkte: die Bedürfnisse der Kinder und Jugendlichen, ihre Lebenssituation sowie den Stand ihrer intellektuellen und sozial-moralischen Entwicklung. Für deren Analyse stützt sich Tulodziecki jeweils auf Entwicklungsmodelle, die durch hierarchische (invariante) Stufen gekennzeichnet sind. Aufgabe des Unterrichts ist dabei immer, Lernprozesse anzuregen und den Schülerinnen und Schülern in allen Bereichen das Erreichen der nächst höheren Entwicklungsstufe zu ermöglichen. Dies ist nach Tulodziecki besonders gut möglich, wenn die Unterrichtsaufgaben problemorientiert gestaltet werden oder es sich um Entscheidungsfälle, Gestaltungs- oder Beurteilungsaufgaben handelt (vgl. ebd., S. 77ff.).

$\mathrm{Zu}$ den auch für die Ausbildung von Medienkompetenz und medienpädagogischer Kompetenz als spezifischen Gegenstandsbereichen wichtigen Unterrichtsprinzipien lässt sich im Einzelnen festhalten, daß Tulodziecki - bezogen auf die Bedürfnisse von Kindern und Jugendlichen - auf den integrativ angelegten bedürfnistheoretischen Ansatz des humanistischen Psychologen Abraham Maslow zurückgreift, den er modifiziert. Danach werden „die bewußt wahrgenommenen Bedürfnisse des täglichen Lebens“ als „Mittel zum Zweck der Befriedigung [...] der sogenannten Grundbedürfnisse“ (Tulodziecki 1992, S. 60) interpretiert. Studierende sollen entsprechende wissenschaftliche Modelle im Rahmen ihres Erwerbs medienpädagogischer Kompetenz kennenlernen, um diesen Aspekt im Unterricht hinreichend berücksichtigen zu können.

Das intellektuelle Urteilsniveau der Schülerinnen und Schüler soll durch Unterricht hinsichtlich der Anzahl der Vorstellungsinhalte, ihrer Detaillierung und der Verknüpfung gefördert werden, um auch eine „mehrdimensionale Betrachtungsweise“ (Tulodziecki 1988, S. 109) zu erreichen. Hier legt Tulodziecki das Theorem der kognitiven Komplexität von Schröder, Driver und Streufert zu Grunde, das er weiterführt, indem er es anders akzentuiert und neu strukturiert. Durch Berücksichtigung entsprechender Modelle soll vermieden werden, daß Aufgaben zu schwierig oder zu einfach formuliert werden und dann nicht entwicklungsfördernd wirken.

Eine zentrale Rolle in Tulodzieckis Überlegungen spielt das sozialmoralische Niveau der Kinder und Jugendlichen, für dessen Bestimmung er auf die Entwicklungstheorie Lawrence Kohlbergs zurückgreift. Tulodziecki geht - wie Kohlberg - davon aus, daß eine Weiterentwicklung des sozial-moralischen Niveaus immer nur zur unmittelbar nächsten Stufe erfolgen kann, mit deren Erreichen eine „grundsätzliche Änderung der Orientierung bzw. Denkformen verbunden“ (Tulodziecki/Aufenanger 1989, S. 10; im Original 
unterstrichen) sei. Konzepte zur Vermittlung von Medienkompetenz müssen so angelegt sein, daß sie - wie Unterricht überhaupt - diese Entwicklung fördern, und Konzepte zum Erwerb medienpädagogischer Kompetenz haben den Erwerb von Kenntnissen über diese Entwicklungsfolge zu berücksichtigen.

In wechselseitiger Verschränkung mit diesen allgemeindidaktischen Überlegungen entwickelt der Paderborner Hochschullehrer Anforderungen an das, was allgemein als „Medienkompetenz“ von Schülerinnen und Schülern bezeichnet wird - einen Begriff, den er zur Beschreibung dieser Kompetenzebene übernimmt. Erst in einem deutlich davon abgegrenzten Schritt erarbeitet Tulodziecki Elemente „medienpädagogischer Kompetenz“, wie er die Fähigkeit der Lehrerinnen und Lehrer umschreibt. Das zentrale Ziel der Medienerziehung wird von Tulodziecki - in Umsetzung der allgemeinen Zielvorstellung von Unterricht - in einem ,sachgerechten, selbstbestimmten, kreativen und sozialverantwortlichen Handeln im Zusammenhang mit Medien“ (Tulodziecki 1997a, S. 120) gesehen. Medienkompetenz kann dabei aus Sicht von Tulodzieckis Handlungsmodell „sowohl als Voraussetzung als auch als prozessbedeutsame Variable und als Zielvorstellung für medienbezogenes Handeln“ (Tulodziecki 1997b, S. 187) begriffen werden und ordnet sich als Zielvorstellung in die obige Definition ein, wird also von ihm in allgemeinbildendem Sinn verwandt (vgl. Tulodziecki 1997f., S. 1). Die einzelnen Fähigkeiten lassen sich in fünf medienerzieherischen Aufgabenbereichen beschreiben (abhängig vom Erscheinungsjahr variieren die Benennung der Aufgabengebiete und ihre Reihenfolge in den einzelnen Publikationen leicht):

1. Auswählen und Nutzen von Medienangeboten (unter Abwägung von Handlungsalternativen),

2. Eigenes Gestalten und Verbreiten von Medienbeiträgen,

3. Verstehen und Bewerten von Mediengestaltungen,

4. Erkennen und Aufarbeiten von Medieneinflüssen sowie

5. Durchschauen und Beurteilen von Bedingungen der Medienproduktion und Medienverbreitung.

Diese Aufgabenbereiche der Medienerziehung sind vor dem Hintergrund zu sehen, daß Medien „in zweifacher Weise in Handlungszusammenhänge eingebunden sein können“ (Tulodziecki 1997c, S. 43), und zwar bei der Nutzung vorhandener Medienangebote (vgl. 1.) und bei der eigenen Gestaltung (vgl. 2.). Um Medien angemessen nutzen und gestalten zu können, ist es notwendig, Kenntnis und Verstehen, Analyse- und Urteilsfähigkeit in Bezug auf die drei am Kommunikationsprozess beteiligten Elemente Sender bzw. Kommunikator (vgl. 5.), Botschaft bzw. Medienprodukt (vgl. 3.) und Empfänger bzw. Rezipient (vgl. 4.) zu erwerben (vgl. Tulodziecki 1997a, S. 142).

Erwerb und Weiterentwicklung eigener Medienkompetenz reichen allerdings nicht aus, um diese Schülerinnen und Schülern vermitteln zu können. Tulodziecki trennt die Ebene der medienpädagogischen Kompetenz deutlich 
von der der Medienkompetenz und formuliert als Aufgabe der universitären Lehrerausbildung:

„Auf die Lehramtsausbildung an der Hochschule kommt die Aufgabe zu, die Studierenden der Lehrämter so zu qualifizieren, daß sie ihre späteren Aufgaben im Umgang mit Medien sowie der mit ihnen verbundenen Informations- und Kommunikationstechnologien angemessen wahrnehmen können“ (Tulodziecki 1995, S. 1).

Die Kompetenzen von Lehrpersonen formulierte Tulodziecki in früheren Veröffentlichungen zunächst auf drei Ebenen, die er in späteren Veröffentlichungen in fünf Aufgabenbereiche der Lehrerausbildung ausdifferenziert. Bei den drei Ebenen handelt es sich zum einen um die bereits angesprochene eigene Medienkompetenz, zum zweiten um die Kompetenz, Lernsituationen zu arrangieren, in denen die Schülerinnen und Schüler Medienkompetenz erwerben können, und schließlich um Kenntnisse, Fertigkeiten und Fähigkeiten, „um medienerzieherische Prozesse im schulischen Kontext in Abstimmung und Kooperation mit Kolleginnen und Kollegen sowie mit außerschulischen Medieneinrichtungen in fächerübergreifender Weise projektorientiert zu planen und zu organisieren“ (Tulodziecki 1994b, S. 23).

Wegen der besonderen Bedeutung für Lehrerinnen und Lehrer arbeitet Tulodziecki die zweite und dritte Ebene medienpädagogischer Kompetenz in vier Teilbereiche aus (vgl. Tulodziecki 1997e, S. 17):

1. die Bedeutung von Medien für Kinder und Jugendliche zu erfassen und im Fachunterricht zu berücksichtigen,

2. die Medienangebote im Unterricht in reflektierter Weise zu verwenden,

3. Medienthemen im Unterricht in angemessener Form zu behandeln,

4. in der Schule personale und institutionelle Bedingungen für medienpädagogische Umsetzungen zu durchschauen.

Die Bedeutung von Medien für Kinder und Jugendliche im Unterricht berücksichtigen zu können beinhaltet, die Studierenden zu sensibilisieren für die medienbezogenen Lernvoraussetzungen auf Seiten der Schülerinnen und Schüler. Hinter diesem Element medienpädagogischer Kompetenz steht u.a. die These, daß sich durch außerschulische Mediennutzung „die kenntnis-, fähigkeits- und motivationsbezogenen Voraussetzungen fiir den Fachunterricht ändern“ (Tulodziecki 1997d, S. 31).

Bezogen auf den Einsatz von Medien im Unterricht sollen die Studierenden lernen, die für die Fächer auf dem Markt befindlichen Medienangebote „nach lernrelevanten Kriterien zu analysieren und auszuwählen“ sowie Konzepte für die Verwendung dieser Medien „im Rahmen weiterentwickelter Lehr- und Lernformen“ zu erarbeiten und diese dann umzusetzen (Tulodziecki 1997e, S. 17). Als Grundlage hierfür ist es wichtig, verschiedene lehr-lerntheoretische Ansätze zu kennen. Denn:

„Medienverwendung beim Lehren und Lernen kann nur so gut sein wie das zugrunde liegende Unterrichtskonzept“ (Tulodziecki 1993). 
Medien im Unterricht zum Thema machen zu können, umfasst den Bereich der Medienerziehung. Die Medienentwicklung hat nach Tulodziecki zur Folge, „daß es Verlagerungen bei den Fachinhalten gibt. Traditionelle Fachinhalte verlieren unter Umständen an Bedeutung, zum Teil bilden sich neue Fachinhalte aus" (Tulodziecki 1997d, S. 32). Den Studierenden sollen diese Veränderungen bewusst sein. Darüber hinaus geht es darum, die Erziehungsund Bildungsaufgaben im Bereich der Medien für die jeweiligen Fächer $\mathrm{zu}$ erkennen und in fachbezogener sowie fächerüberschreitender Weise Umsetzen zu können. Hierfür ist es nach Tulodziecki hilfreich, die verschiedenen Konzepte zur Informationstechnischen Bildung und zur Medienpädagogik zu kennen.

Schließlich gilt es, die in der Schule geltenden Rahmenbedingungen zu beachten, so daß der Erwerb entsprechender Kenntnisse und einer entsprechenden Reflexionsfähigkeit auch ein Element medienpädagogischer Kompetenz darstellt. In den Zielvorstellungen des BIG-Projekts heißt es in diesem Zusammenhang, die Studierenden sollen in der Lage sein,

„a) die Bedeutung der Medien für Fragen der Professionalität des Lehrberufs zu reflektieren,

b) schulische Bedingungen von Medienverwendung, Medienerziehung und informationstechnischer Grundbildung zu bedenken und

c) Ideen für die schulische Umsetzung zu entwickeln“ (Tulodziecki 1996, S. 1).

\section{Kritische Würdigung der beiden Ansätze, Synthese und Schlussfolgerungen}

Eine Gesamtbetrachtung der Ansätze von Baacke und Tulodziecki lässt zentrale Gemeinsamkeiten, aber auch wichtige Unterschiede erkennen. Im Folgenden wird zunächst jeder Ansatz einzeln untersucht, bevor eine Synthese vorgenommen wird und Schlussfolgerungen gezogen werden.

Dieter Baacke arbeitet sehr differenziert aus, was Medienkompetenz in einer kritisch-emanzipatorischen Sicht umfassen kann. Er fundiert ein Leitbild aktiver Mediennutzung, das Ausgang nimmt und sein Ziel findet in mündigen Bürgerinnen und Bürgern und so Chancen und Gefahren der Medienentwicklung in einer modernen Gesellschaft in den Blick bekommt. Wie dieses Konzept realisiert werden kann, bleibt weitgehend offen. Baacke arbeitet weder für die Schule noch für die außerschulische Medienpädagogik systematisch aus, wie ein individueller Erwerb entsprechender Medienkompetenz gefördert werden kann. Entscheidende Leerstellen weisen aus professionstheoretischer Sicht aber v.a. seine Aussagen zur medienpädagogischen Kompetenz auf. Das Transferieren von Medienkompetenz als Grundqualifikation aller Bürgerinnen und Bürger - und damit auch der Schülerinnen und Schüler - auf die Ebene der Lehrerinnen und Lehrer - lediglich ausgeweitet um für diese Personengruppe wichtige Wissensbestände - reicht nicht aus, daß diese die Schülerinnen und Schüler bei einem Erwerb der entsprechenden 
Kompetenz auch unterstützen können. Angesichts dieses Defizits ist nicht verwunderlich, daß Baacke die Schüler- und Lehrer-Ebenen der Medienkompetenz begrifflich nicht trennt. Wichtig erscheint allerdings der aus dem Baackeschen Ansatz zu ziehende Schluss, daß sich die Medienkompetenz auf der Ebene der Schülerinnen und Schüler von der Medienkompetenz als Basiskompetenz der Lehrerinnen und Lehrer in Bezug auf die erforderlichen Wissensbestände durchaus unterscheidet, indem sie für Letztere - im Sinne einer Fähigkeit zur didaktischen Reduktion - umfangreicher sind.

Dieter Baacke hat mit der Verwendung des Kompetenzbegriffs in einem spezifischen Verständnis - einerseits als anthropologische Konstante, andererseits als Ziel eines Entwicklungsprozesses und damit als Norm - Chomsky gegenüber einen wichtigen Perspektivenwechsel vorgenommen, der den Kompetenzbegriff aus seinem biologistischen Ursprung löst und ihn zu einem pädagogischen Begriff macht. Allerdings ist festzustellen, daß von Baacke weder die Verbindung bzw. die Differenz zu Chomsky noch die Aktualisierung des Kompetenzbegriffs im Begriff Medienkompetenz theoretisch genauer geklärt wird. So formuliert z.B. Heydrich:

„Auch für Baackes Überlegungen ist Chomskys Ansatz kaum mehr als ein Sprungbrett“ (Heydrich 1995, S. 226).

Inkonsistent wird das Theoriegebäude Baackes zudem durch wiederholte Widersprüche. Gehört Erziehung aufgrund der normativen Zielstellung quasi systemimmanent zum Kompetenzbegriff Baackes, spricht er sich an mehreren Stellen gegen alle erzieherische Intentionen und Prozesse aus, da diese auf Wirkungen und ein Ziel ausgerichtet seien: „Seit der Aufklärung sind wir uns jedoch einig, daß ein demokratisches Staatswesen Bürger, die über sich selbst verfügen können, benötigt - während Erziehung doch gerade Einordnung und Unterwerfung, wenn auch vielleicht nur für eine bestimmte Zeit, fordert“ (Baacke 1997b, S. 17f.).

Abgesehen davon, daß hier eine sehr traditionelle Auffassung von Erziehung deutlich wird, nimmt Baacke keine umfassende erziehungswissenschaftliche Bestimmung seines theoretischen Ansatzes vor, so daß offen bleibt, auf welche Grundposition er sich bei solchen Aussagen bezieht. Erst in einem seiner letzten Texte (vgl. Baacke 1999a) wird deutlich, daß sich Baacke mit der in diese Richtung gehenden Kritik auseinander setzte - mit der Folge allerdings, daß der Zusammenhang zwischen seinen Einzelarbeiten weniger deutlich wird. Ebenso problematisch erscheint, daß Baacke die drei erziehungswissenschaftlichen Grundbegriffe Erziehung (s.o.), (Medien-)Sozialisation und (Wahrnehmungs-)Bildung nebeneinander verwendet, ohne genauer zu klären, was er jeweils meint, und ohne die Bedeutung gegeneinander abzugrenzen. Dies ist v.a. deshalb zu kritisieren, weil eine allgemeingültige Abgrenzung untereinander in der Erziehungswissenschaft noch immer ein „ungelöstes Problem“ (Vogel 1996, S. 481) darstellt. Schließlich soll in diesem Zusammenhang noch die Verwendung des Begriffs der „Vermittlung“ durch Baacke problematisiert werden, der zum Kompetenzbegriff m.E. in einem gewissen 
Widerspruch steht. Baacke thematisiert nicht, daß eigentlich auch der Bereich der „Vermittlung“ der aktiven (Mit-)Konstruktion bedarf.

Die Stärke des Ansatzes von Tulodziecki liegt demgegenüber darin, daß er die allgemeindidaktische Diskussion umfassender einbezieht und eine analytisch orientierte, auf Lehr-Lernprozesse bezogene Systematik sowohl für die Ebene der Medienkompetenz als auch für die Ebene der medienpädagogischen Kompetenz entwickelt. Beide Kompetenzebenen differenziert er in fünffacher Weise aus, wobei er für die Ebene der Medienkompetenz auch eine theoriegestützte, Gewichtung der Elemente untereinander vornimmt. Dies ergänzend lassen sich auf der Ebene der medienpädagogischen Kompetenz zwei Kernbereiche ausmachen: der Einsatz von Medien als Unterrichtsmittel und die Behandlung von Medien als Unterrichtsgegenstand, für die der Erwerb von Kenntnissen, Fertigkeiten und Fähigkeiten in drei weiteren Kompetenzbereichen notwendig ist. Eine vergleichbare Unterscheidung findet sich auch im BLK-Orientierungsrahmen „Medienerziehung in der Schule“ (vgl. BLK 1995, S. 24f.), an dem Tulodziecki mitgewirkt hat.

Im Unterschied zu Aufenanger (vgl. Aufenanger 1996b, S. 462) folge ich mit dieser Grobstruktur der Überzeugung, daß mediendidaktische Kennmisse, Fertigkeiten und Fähigkeiten in die Konzeption mit einzubeziehen sind. Medienerzieherische allein reichen nicht aus, um medienpädagogische Kompetenz zu erwerben, da sich der Einsatz von Medien im Unterricht immer auch auf die Medienkompetenz der Schülerinnen und Schüler auswirkt, Medienerziehung also nicht losgelöst betrieben werden kann.

Der Ansatz von Tulodziecki überzeugt darüber hinaus zum einen durch einen deutlichen und konzeptionell nachvollziehbaren Bezug auf die Ziele der Schule, wie es für die Lehrerausbildung notwendig ist. Zum anderen sind die zu Grunde liegenden theoretischen Bezüge untereinander vereinbar, da sie im weitesten Sinne dem kognitionstheoretischen Umfeld zugeordnet werden können. Tulodziecki leistet auch eine Umsetzung dieser Konzeption in konkrete Ausbildungsinhalte für Schule und Hochschule, die er fächerübergreifend anlegt, um sowohl „den mangelnden Kenntnissen vieler geistes- und sozialwissenschaftlich orientierter Lehrer zu Fragen der technischen Entwicklung“ als auch der „Hilflosigkeit von Fachlehrern im naturwissenschaftlichen und technischen Bereich [...], wenn es um die Vermittlung individueller und gesellschaftlicher Konsequenzen neuer Technologien geht,“ zu begegnen (Tulodziecki 1985, S. 119). Die Variabilität dieser Art des Herangehens an Medienerziehung macht eine Integration in verschiedene Altersstufen, Fächer und Themen möglich. Dieses Vorgehen beinhaltet gleichzeitig eine Kritik an Vorstellungen, zunächst von fachspezifischen Fragen auszugehen und zu überlegen, welche Rolle Medien spielen können, wie es beispielsweise von Wermke gefordert wird (vgl. Wermke 1996). In konzeptioneller Hinsicht stellen Mediendidaktik und Medienerziehung die notwendigen Aufgaben dar, die in den Unterrichtsfächern als Anwendungsfeldern erfüllt werden müssen. Es handelt sich dabei zwar immer um ein wechselseitiges Verhältnis - aber eben aus dem Blickwinkel medienpädagogischer Erfor- 
dernisse und Zielbestimmung. Für Schule im herkömmlichen Stil gilt sicher, wie Wermke kritisiert, daß fächerübergreifende Vorhaben, insbesondere in Form des Projekts, ungewöhnlich sind und vielleicht sogar eine „Überforderung“ (Wermke 1997, S. 22) einzelner Fachlehrerinnen und Fachlehrer darstellen, aber sie bieten eine Chance zur Erneuerung, die immer dringlicher von allen Seiten gefordert wird.

Tulodziecki leistet darüber hinaus eine erste Erörterung der medienpädagogischen Aufgabe des universitären Studiums im Rahmen der mehrphasigen Lehrerausbildung (vgl. Tulodziecki 1998, S. 137 und Tulodziecki 1999, S. 263ff.): Die Ausbildung an der Universität soll einerseits den Erwerb der wissenschaftlichen Grundlagen für die mediendidaktische, die medienerzieherische, die sozialisationsbezogene und die Schulentwicklungskompetenz ermöglichen und andererseits die eigene Medienkompetenz stärken. Die eher umsetzungsorientierte Ausbildung an den Studienseminaren sollte dann die unterrichtliche Umsetzung mediendidaktischer und medienerzieherischer Vorhaben und die Berücksichtigung entsprechender Lernvoraussetzungen in den Mittelpunkt stellen. Die Lehrerfortbildung als dritte Phase schließlich könne dann einen Akzent auf die Gestaltung der Rahmenbedingungen medienpädagogischen Handelns legen und zugleich dazu dienen, regionale Beratungs- und Unterstützungssysteme aufzubauen.

Unabhängig von der Kritik in Einzelpunkten, widerlegen Baacke und Tulodziecki die gemeinhin formulierten Thesen, die Konzepte für die Lehrerausbildung seien zu technisch orientiert, sähen die Jugendlichen nur als passive Objekte oder vernachlässigten eine Werteerziehung. Beide Autoren gehen von Medien als Unterstützer eines Kommunikationsprozesses aus, entwickeln eine normative Zielstellung und setzen auf handlungsorientierte Unterrichtskonzepte. Baacke und Tulodziecki lösen damit auch die Forderungen der Ständigen Konferenz der Kultusminister ein, die der Schule als Aufgabe zuschreiben will, den Medienumgang ,verantwortlich, kritisch, aber auch kreativ werden zu lassen und weiterzuentwickeln“, und die sich eine Medienpädagogik wünscht, die „von einer grundsätzlichen Offenheit gegenüber der Medienwelt“ ausgeht und die die „z.T. disparaten Erfahrungen und Handlungsmuster der Heranwachsenden im Umgang mit Medien“ berücksichtigt, und zwar mit „angemessenen Unterrichtsmethoden bzw. Arbeitsformen“ (KMK 1995, S. Iff.). Die aktuellen Konzepte haben sich damit weit entfernt von früheren medienpädagogischen Zielen, die von bewahrpädagogischen über kontrollorientierte bis zu ideologiekritischen Ansätzen reichten. Diese traditionellen Ansätze könnten der Verwendung des Kompetenzbegriffs auch nicht gerecht werden, da sie auf der Performanzebene verbleiben. Weder Verbote bzw. Vorenthalten bestimmter Medienangebote noch - ausschließlich - kritische Analyse ermöglichen selbstverantwortliches Handeln in neuen unbekannten Situationen. Das bedeutet allerdings nicht, daß diese Aspekte keine Rolle mehr spielen, sondern sie wurden angemessen in die gegenwärtigen Ansätze integriert. So stellen die Medienpädagogen Jürgen Hüther und Bernd Schorb zu Recht fest, daß sich Ziele wie „Bewahren“, „Informieren“ 
oder „Sensibilisieren“ in unterschiedlicher Gewichtung noch in allen medienpädagogischen Positionen - nicht nur in den beiden ausgewählten - finden (vgl. Hüther/Schorb 1997, S. 249).

Baacke und Tulodziecki reagieren auch auf Kritik, die das Fehlen einer pädagogischen Theorie für die Medienerziehung (vgl. Merkert 1996, S. 1059ff.) monieren und das Fehlen von Angaben, welche Qualifikationen Lehrpersonen erwerben müssen, um Schülerinnen und Schülern einen angemessenen Umgang mit Medien vermitteln zu können (vgl. Sobiech 1997, S. 51). Die obige Darstellung hat gezeigt, daß Baacke und Tulodziecki - wenn auch in unterschiedlicher Weise - Aussagen hierzu machen.

Auffällig ist allerdings, wie isoliert die Ansätze von Baacke und Tulodziecki nebeneinander stehen. Bezüge auf die Konzepte des jeweils anderen Autors finden sich nur sehr selten. Damit werden Chancen einer fundierten Weiterentwicklung vergeben. Offensichtlich gilt dieses Manko einer ungenügenden gegenseitigen Rezeption für weite Teile der medienpädagogischen Literatur, der Sobiech einen „additiven Charakter“ (Sobiech 1997, S. 22) bescheinigt. Wolfgang H. Swoboda sieht die Ursache hierfür darin, daß es keine historischen Darstellungen medienpädagogischer Theorien und Konzepte gibt mit der Folge:

„Gemeinsame Traditionslinien und komplementäre Ansätze bleiben oftmals unentdeckt und ungenutzt“ (Swoboda 1994, S. 21).

Aus Arbeiten im weiteren Umfeld der Medienpädagogik lassen sich zwei wichtige Aspekte ergänzen, die weder bei Baacke noch bei Tulodziecki Berücksichtigung finden: Schulz-Zander setzt für die Informationstechnische Grundbildung (ITG) einen starken Akzent auf die Berücksichtigung der Geschlechterverhältnisse als Thema der Lehrerausbildung. Verstärkt Lehrerinnen - als Vorbilder - zu qualifizieren und die Studierenden als zukünftige Lehrerinnen und Lehrer in Bezug auf die Geschlechterfrage zu sensibilisieren, sieht sie angesichts des zur Zeit vorwiegend von Männern dominierten Gegenstandsbereichs als zentrale Aufgaben der Lehrerausbildung an (vgl. Schulz-Zander 1992, S. 219). Schulz-Zander macht als Ursache der Distanz der Mädchen zur ITG eine doppelte Auswirkung der gesellschaftlichen Strukturen, insbesondere der geschlechtsspezifischen Arbeitsteilung aus. Beeinflusst hierdurch prägen sich zum Einen entsprechende Einstellungen und Verhaltensweisen im Laufe des familiären, schulischen und außerschulischen Sozialisationsprozesses aus mit der Folge, daß Mädchen und Jungen unterschiedliche Selbstkonzepte entwickeln (vgl. Schulz-Zander 1989, S. 164). Für die Mädchen bedeutet dies fast quasi automatisch eine Technikferne. Schulz-Zander:

„Sich in männlich besetzten Bereichen als kompetent zu erweisen hieße gerade, den für Mädchen und Frauen vorgezeichneten Weg zur Erhöhung des Selbstwertgefühls zu verlassen“ (Schulz-Zander 1988, S. 12).

Aufgabe der Lehrerinnen und Lehrer ist es laut Schulz-Zander in diesem Zusammenhang, den Schülerinnen und Schülern „eine Unterstützung bei der 
Überschreitung gesellschaftlich festgelegter Rollen, bei der Veränderung des Selbstkonzepts zu geben“ (Schulz-Zander 1989, S. 166). Im Rahmen der Lehrerausbildung sollen die Lehrerinnen und Lehrer befähigt werden, die Geschlechterproblematik im Unterricht zu berücksichtigen, und zwar sowohl als eigenes Thema als auch immanent, wenn es etwa um eine „Entmystifizierung“ (ebd., S. 211) der Informationstechnik geht. Die Bund-Länder-Kommission für Bildungsplanung und Forschungsförderung weist in diesem Zusammenhang auch auf die Notwendigkeit einer Sensibilisierung für den Umgang mit Vorbehalten seitens der Elternhäuser hin (vgl. BLK 1987, S. 29).

Von Hauf-Tulodziecki kommt ebenfalls aus der Sicht der ITG eine weitere Anregung: Deren fast ausschließlicher Bezug auf die Arbeitswelt wurde von Medienpädagogen zu Recht kritisiert, der aber bei einer Verbindung der ITG mit der Medienerziehung wieder Bedeutung erhält. Hauf-Tulodziecki:

„In der traditionellen Medienerziehung wurden elektronische Medien in erster Linie im Hinblick auf ihren Einfluss auf private Lebensbereiche und das Freizeitverhalten als bedeutsam angesehen. In einer erweiterten Medienerziehung sollen Medieneinflüsse auch auf die Arbeitswelt und auf Formen der beruflichen Kommunikation betrachtet werden“" (Hauf-Tulodziecki 1995, S. 14).

Dieser Bezug ergänzt die Frage nach den Medieneinflüssen auf das Individuum und ihrer Verarbeitung durch dieses, auf die Baacke nur wenig eingeht. Die Reflexivität, die er im Bereich der Medienkritik auflistet, beschränkt sich darauf, daß die Rezipientinnen und Rezipienten ihre Stellung in der Medienwelt erkennen und daraus medienkritische Schlussfolgerungen ziehen. Tulodziecki weist hier auf die Notwendigkeit hin, sich individuelle medienbedingte Emotionen, Vorstellungen und Verhaltensorientierungen bewusst zu machen und sie überprüfen zu können. Dies gilt auch - oder gerade - für die Person des Lehrers bzw. der Lehrerin selbst. Baackes medienkritische Intention deutet dafür auf eine andere Akzentsetzung hin, die Herzig mit seiner Formulierung „Verbreitung und Einfluss von Medien auf Individuum und Gesellschaft“ (Herzig 1997, S. 51; Hervorhebung nicht im Original) als Oberbegriff einer Synthese verschiedener medienpädagogischer Ansätze, u.a. auch der von Baacke und Tulodziecki, berücksichtigt.

Auf der Basis dieser Bewertungen und Ergänzungen kann nun eine Synthese vorgenommen werden. Der in weiten Teilen überzeugende Ansatz von Tulodziecki soll im Folgenden als Grundlage und Ausgangspunkt gewählt werden. Medienpädagogische Kompetenz setzt sich danach aus fünf Teilkompetenzen zusammen:

- aus einem ersten Kernbereich, der mediendidaktischen Kompetenz: als Fähigkeit von Lehrerinnen und Lehrern zur reflektierten Verwendung von Medien und Informationstechnologien in geeigneten Lehr- und Lernformen und deren Weiterentwicklung,

- aus einem zweiten Kernbereich, der medienerzieherischen Kompetenz: als Fähigkeit von Lehrerinnen und Lehrern, Medienthemen im Sinne pädagogischer Leitideen im Unterricht behandeln zu können, 
- aus einer Voraussetzung, um diese beiden Kernbereiche in der Schule angemessen wahrnehmen zu können, der sozialisationsbezogenen Kompetenz im Medienzusammenhang: als Fähigkeit von Lehrerinnen und Lehrern zur konstruktiven Berücksichtigung der medienbedingten Lernvoraussetzungen der Schülerinnen und Schüler,

- aus einer ebenfalls auf beide Kernbereiche bezogenen Teilkompetenz, der Schulentwicklungskompetenz im Medienzusammenhang: als Fähigkeit von Lehrerinnen und Lehrern zur innovativen Gestaltung der Rahmenbedingungen medienpädagogischen Handelns in der Schule sowie

- schließlich aus einer Basiskompetenz, die alle Bürgerinnen und Bürger besitzen und die die Studierenden damit schon vor Studienbeginn in der Schulzeit oder durch außerschulische Aktivitäten erworben haben sollten, der eigenen Medienkompetenz von Lehrerinnen und Lehrern: als Fähigkeit zu eigenem sachgerechten, selbstbestimmten, kreativen und sozialverantwortlichen Handeln im Zusammenhang mit Medien und Informationstechnologien.

Diese fünf Teilkompetenzen lassen sich weiter ausdifferenzieren, so daß man ein umfassendes Konzept für die Gestaltung einer medienpädagogisch angemessenen Lehrerausbildung erhält (vgl. Blömeke 2000, S. 148ff.). Forderungen nach dem bloßen Erwerb von Internet-Führerscheinen im Studium erscheinen in dieser Sichtweise als kurzschlüssig, da der Erwerb einer komplexen medienpädagogischen Kompetenz einen langfristigen Prozess darstellt.

Daß dennoch Erwartungen im instrumentell-qualifikatorischen Sinne an die Lehrerausbildung formuliert werden, liegt vermutlich an der Verstärkung studentischer Interessen (häufig fehlende eigene Medienkompetenz aufgrund einer in diesem Punkt noch defizitären schulischen Ausbildung) durch einen falschen ,Respekt' (= Schwellenangst) auf Seiten vieler Hochschullehrender vor der neuen, ungewohnten und daher überschätzten Thematik sowie an ökonomisch-politischen Interessen der Landesregierungen (Sorge um auf Dauer mangelhafte Qualifikation der Lehrerinnen und Lehrer in diesem Bereich, damit einer dauerhaft mangelhaften Qualifikation der Schülerinnen und Schüler mit der Gefahr, als Land ökonomisch ,abgehängt' zu werden). In Nordrhein-Westfalen wird diese Tendenz an vielen aktuellen Vorgängen deutlich: an der Gründung einer „e-nitiative“ mit einem Internet-Führerschein als wichtigem Ziel, ohne den zukünftig keine Lehrerin bzw. kein Lehrer mehr eingestellt werden soll (vgl. http://www.e-nitiative.nrw.de 2000); an der Anerkennung mancher Lehrveranstaltung als einen regulären Studienbestandteil, obwohl es sich um nichts anderes ist als eine Einführung in die Internet-Benutzung handelt etc. Wie problematisch diese Entwicklung ist, wird möglicherweise durch einen Vergleich mit Kenntnissen in Sprachen wie Englisch, Französisch und Latein deutlicher, die für eine Reihe von Lehramtsstudiengängen Voraussetzung für die Aufnahme des Hauptstudiums sind (z.B. in Deutsch, Geschichte, Pädagogik und Religionslehre; wird eine Fremdsprache studiert, muss es sich um Kenntnisse in anderen Fremdsprachen als der studierten Sprache handeln): Da es sich 
bei diesen Sprachkenntnissen um einen klassischen Bestandteil von Allgemeinbildung handelt, wird nicht nur von Seiten der Hochschulen, sondern auch von Seiten der Landesregierung als rahmensetzender Instanz erwartet, daß entsprechende Sprachkenntnisse der Studierenden entweder aus der Schulzeit resultieren oder von ihnen parallel zum Studium erworben werden (vgl. MSWWF 1998, S. 9f. und 50). An keiner Stelle stand bisher zur Diskussion, Sprachkurse als Teil eines wissenschaftlichen Studiums anzurechnen.

Um die notwendigen Voraussetzungen für die zentrale Auseinandersetzung mit Medienerziehung und Mediendidaktik im Lehramtsstudium zu schaffen, muss die Hochschule allerdings die Chance dazu einräumen, daß Defizite ausgeglichen werden können, die bei den Erstsemestern typischerweise vorliegen - und dazu gehört die Stärkung der eigenen Medienkompetenz. In zahlreichen Angeboten sollte Studierenden also die Chance gegeben werden, parallel zum Studium die eigenen Kenntnisse, Fertigkeiten und Fähigkeiten im Medienzusammenhang weiterzuentwickeln. Ließe sich indes als eigentlicher Standard in der Lehrerausbildung der Erwerb von medienpädagogischer Kompetenz im obigen Sinne realisieren, könnte sich Schule mit Recht als den Herausforderungen der Informationsgesellschaft gewachsen ansehen.

\section{Literatur}

Aufenanger, St. (a): Die neuen Medien und die Pädagogik. Tendenzen in der Medienpädagogik. In: Bildung und Erziehung, 49 (1996) 4, S. 449-460

Aufenanger St. (b): Zur Zusammenarbeit von Elternhaus, Schule und Hochschule in der Medienerziehung. In: Pädagogik und Schulalltag, 51 (1996) 4, S. 460-470

Baacke, D. (a): „Medienkompetenz“: theoretisch erschließend und praktisch folgenreich. In: merz, 43(1999) 1, S. 7-12

Baacke, D. (b): Was ist Medienkompetenz? Fünf Statements zu einem facettenreichen Begriff. In: Schell, F./Stolzenburg, E./Theunert, H. (Hrsg.): Medienkompetenz: Grundlagen und pädagogisches Handeln. München 1999, S. 19-20

Baacke, D. (a): Medienpädagogik. Tübingen 1997

Baacke, D. (b): Kevin, Wayne und andere - Kinder und ästhetische Erfahrung. Medienkompetenz: Chance für Qualifikationen und Qualitäten. In: Gottberg, J. von/Mikos, L./ Wiedemann, D. (Hrsg.): Kinder an die Fernbedienung. Konzepte und Kontroversen zum Kinderfilm und Kinderfernsehen. Berlin 1997, S. 13-21

Baacke, D. (c): Diskurs der Informationsgesellschaft. In: Enquete-Kommission „Zukunft der Medien in Wirtschaft und Gesellschaft - Deutschlands Weg in die Informationsgesellschaft“/Deutscher Bundestag (Hrsg.): Medienkompetenz im Informationszeitalter. Bonn 1997, S. 23-27

Baacke, D.: „Gesamtkonzept Medienkompetenz“. Der Medienpädagoge Dieter Baacke über die Konjunktur (s)eines Begriffs. In: agenda. Zeitschrift für Medien, Bildung, Kultur, 23 (MärzApril 1996), S. 12-14

Baacke, D.: Projektbeschreibung. Medienkompetenz in der Lehrerbildung (MeKoLa). Bielefeld 1995

Baacke, D.: Sprachlose Bürger? Medienkompetenz als zentrales Ziel von Medienpädagogik. In: Wunden, W. (Hrsg.): Öffentlichkeit und Kommunikationskultur. Hamburg 1994, S. 231-243 
Baacke, D. (a): Handlungsorientierte Medienpädagogik. In: Schill, W./Tulodziecki, G./Wagner, W.R. (Hrsg.): Medienpädagogisches Handeln in der Schule. Opladen 1992, S. 33-58

Baacke, D. (b): Theorie der Medienpädagogik. In: Burkart, R./Hömberg, W. (Hrsg.): Kommunikationstheorien. Ein Textbuch zur Einführung. Wien 1992, S. 171-190

Baacke, D.: Zum ethischen Orientierungsrahmen der Medienpädagogik. In: Issing, Ludwig J. (Hrsg.): Medienpädagogik im Informationszeitalter. Weinheim 1987, S. 53-71

Baacke, D.: Kommunikation und Kompetenz. Grundlegung einer Didaktik der Kommunikation und ihrer Medien. München 1973

Blömeke, S.: Medienpädagogische Kompetenz. Theoretische und empirische Fundierung eines zentralen Elements der Lehrerausbildung. München 2000

Bund-Länder-Kommission für Bildungsplanung und Forschungsförderung: Medienerziehung in der Schule. Orientierungsrahmen (= BLK-Materialien zur Bildungsplanung und zur Forschungsförderung; 44). Bonn 1995. http://www.iid.de/schule/sonstiges/heft44.html (Zitierung laut Ausdruck v. 31.07.1997)

Bund-Länder-Kommission für Bildungsplanung und Forschungsförderung: Gesamtkonzept für die informationstechnische Bildung (= BLK-Materialien zur Bildungsplanung; 16). Bonn 1987

Dewe, B./Ferchhoff, W./Radtke, F.-O.: Einleitung. Auf dem Wege zu einer aufgabenzentrierten Professionstheorie pädagogischen Handelns. In: Dewe, B./Ferchhoff, W./ Radtke, F.-O. (Hrsg.): Erziehen als Profession. Zur Logik professionellen Handelns in pädagogischen Feldern. Opladen 1992, S. 7-20

Hauf-Tulodziecki A.: Handlungsorientiert, fächerübergreifend. Medienerziehung und informationstechnische Grundbildung. In: medien praktisch, 19 (1995) 4, S. 13-16 Herzig, B.। Arbeitsgruppe „Erziehungswissenschaft“. In: Tulodziecki, G./Blömeke, S. (Hrsg.): Neue Medien - Neue Aufgaben für die Lehrerausbildung. Tagungsdokumentation. Gütersloh 1997, S. 39-54

Heydrich, W.: Nachträgliches zur Kompetenz. In: Lauffer, J./Volkmer, I. (Hrsg.): Kommunikative Kompetenz in einer sich verändernden Medienwelt (= Schriftenreihe der Gesellschaft für Medien und Kommunikationskultur in der Bundesrepublik; 9). Opladen 1995, S. 223-234

http://www.e-nitiative.nrw.de. 2000

Hüther, J./Schorb, B.: Medienpädagogik. In: Hüther, J./Schorb, B./Brehm-Klotz, Ch. (Hrsg.): Grundbegriffe Medienpädagogik. München 1997, S. 243-252

Hugger, K.-U. (a): Medienkompetenz für Lehrerstudenten. Zum Bielefelder Modellprojekt Medienkompetenz in der Lehrerausbildung. In: medien praktisch, 19 (1995) 4, S. 16-19

Hugger, K.-U. (b): Zwischen Schülerzeitung und Multimedia. Das Modellprojekt „Medienkompetenz in der Lehrerausbildung“ an der Universität Bielefeld und sein Kontext. In: FWU (Institut für Film und Bild in Wissenschaft und Unterricht) Magazin, 7 (1995) 5, S. 48-51

KMK/Sekretariat der Ständigen Konferenz der Kultusminister der Länder in der Bundesrepublik Deutschland (Hrsg.): Medienpädagogik in der Schule. Erklärung der Kultusministerkonferenz vom 12.05.1995 mit Übersicht über wichtige medienpädagogische Aktivitäten in den Ländern. Bonn 1995

Kubier, H.-D.: Medienkompetenz - Dimensionen eines Schlagwortes. In: Schell, F./Stolzenburg, E./ Theunert, H. (Hrsg.): Medienkompetenz: Grundlagen und pädagogisches Handeln. München 1999, S. 25-47

Merkert, P. R. : Medienpädagogik. In: Hierdeis, H./Hug, T. (Hrsg.): Taschenbuch der Pädagogik. Bd. 3: Gerontagogik - Organisation. Hohengehren 1996, S. 1057-1066

Ministerium für Schule und Weiterbildung, Wissenschaft und Forschung des Landes Nordrhein-Westfalen (Hrsg.): Lehrerausbildung. Teil 1: Studium. Frechen 1998 
Schulz-Zander, R.: Für die Gleichstellung von Mädchen und jungen Frauen in der Informationstechnischen Bildung. Empfehlungen einer IPN-Curriculum-Konferenz. In: Glumpler, E. (Hrsg.): Mädchenbildung - Frauenbildung. Beiträge der Frauenforschung für die Lehrerinnenbildung. Bad Heilbrunn/Obb. 1992, S. 210-220

Schulz-Zander, R.: Konzepte und Strategien zur informationstechnologischen Bildung für Mädchen und junge Frauen. In: Schelhowe, H. (Hrsg.): Frauenwelt - Computerräume. Fachtagung - Proceedings. Berlin u.a. 1989, S. 164-174

Schulz-Zander, R.: Mädchenbildung und Neue Technologien. Eine Synopse. In: LOG IN. Informatik und Computer in der Schule (Berlin) 8 (1988) 1, S. 10-15

Sobiech D.: Theorie und Praxis der Medienerziehung im Vergleich. Eine Analyse von Konzepten, Strukturen und Bedingungen. München 1997

Swoboda, W. H.: Medienpädagogik. Konzeptionen, Problemhorizonte und Aufgabenfelder. In: Hiegemann, S./Swoboda, W. H. (Hrsg.): Handbuch der Medienpädagogik. Theorieansätze - Traditionen - Praxisfelder - Forschungsperspektiven. Opladen 1994, S. 11-24

Tulodziecki, G.: Medienpädagogik in Schule und Lehrerbildung. In: Risse, E./Schmidt, H.- J. (Hrsg.): Von der Bildungsplanung zur Schulentwicklung. Rainer Brockmeyer zu Ehren. Neuwied/Kriftel 1999, S. 252-265

Tulodziecki, G.: Neue Medien als Herausforderung für Schule und Lehrerbildung. In: Kubicek, H. (Hrsg.): Lernort Multimedia. Heidelberg 1998, S. 130-138

Tulodziecki, G. (a): Medien in Erziehung und Bildung. Grundlagen und Beispiele einer handlungsund entwicklungsorientierten Medienpädagogik. Bad Heilbrunn/Obb. 1997

Tulodziecki, G. (b): Erziehung und Bildung im Medienzusammenhang. Ziele, Bedingungen, Aufgaben und Kompetenzen. In: Gottberg, J. von/Mikos, L./Wiedemann, D. (Hrsg.): Kinder an die Fernbedienung. Konzepte und Kontroversen zum Kinderfilm und Kinderfernsehen. Berlin 1997, S. $175-188$

Tulodziecki, G. (c): Medienerziehung als Aufgabe des Deutschunterrichts. In: Erlinger, H. D. (Hrsg.): Neue Medien - Edutainment - Medienkompetenz. Deutschunterricht im Wandel. München 1997, S. 39-51

Tulodziecki, G. (d): Neue Medien und Lehrerausbildung. Überlegungen zum medienpädagogischen Rahmen für die Arbeitsgruppen. In: Tulodziecki, G./Blömeke, S. (Hrsg.): Neue Medien - Neue Aufgaben für die Lehrerausbildung. Tagungsdokumentation. Gütersloh 1997, S. 29-37

Tulodziecki, G. (e): Erziehung und Bildung im Medienzusammenhang: Ziele, Bedingungen, Aufgaben und Kompetenzen. Arbeitspapier. Paderborn 1997

Tulodziecki, G. (f): Medienkompetenz als Ziel schulischer Medienpädagogik. Vortrag im Rahmen der Fachtagung „Schulen ans Netz - eine erste Zwischenbilanz“. Köln 1997 (Ms.)

Tulodziecki, G.: Zielvorstellungen für Lehrveranstaltungen im Teilprojekt „BIG-Lehramtsstudium“. Leicht modifizierte Fassung der Zielvorstellungen gemäß Anlage zum Vertrag. Paderborn 1996 (Ms.)

Tulodziecki, G.: BIG - Bildungswege in der Informationsgesellschaft. Projektskizze zum Teilbereich „Lehramtsstudium“. Paderborn 1995 (Ms.)

Tulodziecki, G. (a): Unterricht mit Jugendlichen. Eine handlungsorientierte Didaktik mit Unterrichtsbeispielen. Bad Heilbrunn/Hamburg 1994

Tulodziecki, G. (b): Prinzipien und Aufgabenbereiche der Medienerziehung. Konsequenz für Kompetenzen von Lehrpersonen. In: Pädagogische Führung, 5 (1994) 1, S. 21-23 Tulodziecki, G.: Erst über Unterricht nachdenken. In: Otto, G. (Hrsg.): Unterrichtsmedien. Seelze 1993, S. 34

Tulodziecki, G.: Mediennutzung als situations-, bedürfnis- und entwicklungsbezogene Handlung Konsequenzen für die Medienerziehung. In: Schill, W./Tulodziecki, G./ Wagner, W.-R. (Hrsg.): Medienpädagogisches Handeln in der Schule. Opladen 1992, S. 59-72 
Tulodziecki, G.: Medienpädagogische Konsequenzen aus der Entwicklung der neuen Informationstechniken. In: Armbruster, B./Kübler, H.-D. (Hrsg.): Computer und Lernen. Medienpädagogische Konzeptionen. Opladen 1988, S. 106-118

Tulodziecki, G.: Medienpädagogische Konsequenzen aus der Entwicklung im Bereich der Informations- und Kommunikationstechniken. In: Medien - Kultur - Bildung. Grünewald 1985, S. 117126

Tulodziecki, G./Aufenanger, St.: Praxis Medienerziehung. Medienethische Reflexionen. Stuttgart 1989

Tulodziecki, G./Schöpf K.: Zur Situation der schulischen Medienpädagogik in Deutschland: Konzepte, Materialien, Praxis und Probleme. In: Bertelsmann Stiftung (Hrsg.): Medienkompetenz als Herausforderung an Schule und Bildung. Ein deutsch-amerikanischer Dialog. Kompendium zu einer Konferenz der Bertelsmann Stiftung. Gütersloh 1992, S. 104-176

Vogel, P.: Scheinprobleme in der Erziehungswissenschaft: Das Verhältnis von „Erziehung“ und „Sozialisation“. In: Zeitschrift für Pädagogik, 42 (1996) 4, S. 481-490

Vollbrecht, R.: Medienkompetenz als kommunikative Kompetenz. Rückbesinnung und Neufassung des Konzepts. In: merz, 43 (1999) 1, S. 13-18

Wagner, W.-R.: Ein umfassendes Medienverständnis. Voraussetzungen für die Integration von Medienerziehung und informationstechnischer Bildung. In: LOG IN. Informatik und Computer in der Schule, 16 (1996) 3, S. 10-14

Wermke, J.: Integrierte Medienerziehung im Fachunterricht. Schwerpunkt: Deutsch. München 1997

Wermke, J.: Medienpädagogik und Fachdidaktik. In: Deutschunterricht, 49 (1996) 9, S. 440-450 